\title{
Significance of atrophy on endometrial sampling in women younger than fifty years of age
}

\begin{abstract}
Objective: To determine the prevalence and significance of endometrial sampling showing atrophy among women younger than 50 years.

Study design: Retrospective study of consecutive endometrial sampling results among women younger than 50 years collected over 2 years. Data were abstracted on patient demographics and clinico-pathological factors.

Results: Among 2,034 eligible women, 96 had atrophy (prevalence 4.7\% [95\% CI: 3.8$5.6 \%]$ ). The corresponding endometrial echo complex was $6.4 \pm 3.6 \mathrm{~mm}$ on transvaginal ultrasound. $93 \%$ of patients had a clinical history compatible with endometrial atrophy, including use of progestin-containing hormones, menopause, or a thin endometrial echo complex on ultrasound; $7 \%$ of patients with abnormal uterine bleeding had ultrasound findings inconsistent with endometrial atrophy, such as an intracavitary lesion or an unevaluable or thickened $(>14 \mathrm{~mm})$ endometrial echo complex. One patient was found to have a uterine malignancy.

Conclusion: Nearly $5 \%$ of women younger than 50 years of age had endometrial atrophy. Of those, $1 \%$ had an underlying malignancy. If a premenopausal woman is not on progestin or combination hormone therapy and ultrasound findings are inconsistent with atrophy, an endometrial sampling result showing atrophy cannot reliably rule out malignancy. We recommend that this subset of patients undergo further testing before malignancy is excluded.
\end{abstract}

Keywords: endometrial atrophy, abnormal uterine bleeding, endometrial sampling, uterine malignancy, premenopausal, endometrial echo complex
Volume 3 Issue 3 - 2015

\author{
Laurie Brunette, I,2 Lirona Katzir, I,2 Malaika \\ Amneus, I,2 Chisa Aoyama,3,4 Christine H \\ Holschneiderl,2 \\ 'Department of Obstetrics and Gynecology, Olive View-UCLA \\ Medical Center, USA \\ ${ }^{2}$ Department of Obstetrics and Gynecology, David Geffen \\ School of Medicine at UCLA, USA \\ ${ }^{3}$ Department of Pathology and Laboratory Medicine, Olive \\ View-UCLA Medical Center, USA \\ ${ }^{4}$ Department of Pathology and Laboratory Medicine, David \\ Geffen School of Medicine at UCLA, USA
}

\section{Correspondence: Christine H Holschneider, Olive}

View-UCLA Medical Center, Department of Obstetrics and

Gynecology, I4445 Olive View Drive, Sylmar, CA 91342, Room 6D-I|6, Tel 8|8-364-3222, Fax 8|8-364-3255,

Email:CHolschneider@dhs.lacounty.gov

Received: October 29, 2015 | Published: December I, 2015

\section{Introduction}

Most women (90\%) with endometrial cancer develop symptomatic vaginal bleeding or discharge, and this is often what prompts them to seek gynecologic care. ${ }^{1}$ The American College of Obstetricians and Gynecologists (ACOG) recommends endometrial tissue sampling in any woman with abnormal uterine bleeding who is older than 45 years, or in younger patients who have failed medical management, have persistent abnormal uterine bleeding, or have unopposed estrogen exposure, such as in obesity or polycystic ovary syndrome. ${ }^{2}$ There is currently a large body of evidence to guide gynecologists in the treatment of women found to have endometrial hyperplasia or cancer on biopsy or curettage. ${ }^{3-5}$ However, there is little to no evidence to guide clinicians in the interpretation of non-hyperplastic, non-neoplastic endometrial sampling findings, which may result in delayed diagnosis in those patients with a false negative result.

One of these seemingly benign results is atrophy of the endometrium, which occurs as a consequence of the prolonged absence of endogenous or exogenous estrogenic stimulation. This is a physiologic occurrence in postmenopausal women, and is the most common cause of postmenopausal vaginal bleeding. ${ }^{6}$ Pathology specimens show a thin mucosa, a decrease in the number of glands and volume of stroma, and absence of nuclear stratification, mitotic activity, and secretory products. ${ }^{7,8}$ Atrophic endometrial glands are also encountered in biopsy specimens of premenopausal patients in the setting of exogenous hormone use. Hormone preparations containing both estrogen and progestin typically result in weak or poorly developed secretory endometrium. Progestin-only compounds result in atrophic glands with minimal or absent mitotic activity, but unlike endometrium devoid of hormonal stimulation, there is also evidence of stromal expansion and pseudodecidualization. The intensity of the histological response will depend upon the potency, dosage, and duration of use of the progestin but this response is similar whether the progestin is delivered via tablets, injections, or an intrauterine device. ${ }^{9-11}$

While atrophic glands are characteristic of premenopausal patients taking exogenous hormones, atrophy in any other setting in a premenopausal woman is an unexpected finding and its significance is not well understood. The minority of uterine cancers can arise in a background of atrophy. These cancers are usually estrogenindependent, less differentiated, and associated with a poorer prognosis than estrogen-dependent tumors. Although endometrial cancer is most commonly encountered in postmenopausal patients, $8.5-14.2 \%$ are diagnosed in premenopausal women. ${ }^{12}$

There are no studies to date that evaluate the risk of malignancy in young women with endometrial atrophy on sampling, and little data exist to direct clinical management in this setting. Few studies have even defined the prevalence of atrophic endometrium in young women. In one small study of 230 premenopausal women with irregular uterine bleeding who underwent endometrial sampling, 8.7\% were found to have atrophy. ${ }^{13}$ In another study of 12,949 endometrial biopsies performed in patients undergoing infertility workup, 2.4\% revealed atrophy. ${ }^{14}$ The objective of this study was to determine the prevalence and clinical significance of endometrial sampling showing atrophy among women younger than 50 years of age. 


\section{Materials and methods}

A retrospective study of women younger than 50 years of age with atrophy on endometrial sampling was conducted at Olive View-UCLA Medical Center, a university-affiliated public safety net hospital. IRB approval was obtained from the Olive View-UCLA Education and Research Institute. The pathology database was searched for consecutive endometrial sampling specimens collected between January 1, 2009 and December 31, 2010 from women less than 50 years of age. Amongst these, the subset that showed endometrial atrophy on pathology was identified. Patients with a previous history of endometrial cancer were excluded. Medical records were reviewed to determine the following for each patient at the time of sampling: age, ethnicity, body mass index, gravity, parity, menopausal status, medical comorbidities, personal and family history of cancer, abnormal uterine bleeding patterns, exogenous hormone use, indication for sampling, pelvic ultrasound findings, and all previous and subsequent uterine or endometrial pathology results. The ultrasound that was performed closest to the date of sampling was included, regardless of whether it occurred before or after the endometrial sampling. However, since ultrasonographic findings of the endometrium are strongly influenced by hormonal factors, data were included in the analysis of the endometrial echo complex thickness only if there was no intercurrent change in hormonal medications between the two procedures. Pathology was assessed for evidence of hormone effect. All slides were re-reviewed by the study pathologist if the original pathology report was equivocal or did not comment on hormone effect.

The prevalence of endometrial samplings showing atrophy among women younger than 50 years of age was calculated. The t-test or one-way ANOVA were used for paramentric variables and the MannWhitney U or Kruskal-Wallis test were used for non-parametric testing of continuous variables as indicated. The Chi-square test was used for categorical variables. All tests were two-sided and a p-value of $<0.05$ was considered significant.

\section{Results}

Over the two-year study period, 2,034 women younger than 50 years of age underwent endometrial sampling with 96 of these women showing atrophic endometrium on pathology. This resulted in a prevalence of endometrial atrophy of $4.7 \%$ (95\% CI: $3.8-$ $5.6 \%)$ in this age group. The majority of pathology specimens, 92 , were from an office endometrial sampling and four were from an endometrial curettage. Figure 1 summarizes the study cohort. Twentythree patients were postmenopausal at the time of sampling. Of the 73 premenopausal patients, 44 were using exogenous hormones. Thirty-four were on a progestin-only regimen and 10 were taking a combination of estrogen and progestin. The indications for hormone use in these patients included a history of endometrial hyperplasia $(n=20)$, irregular bleeding without a history of hyperplasia $(n=21)$, hormonal contraception $(\mathrm{n}=2)$, and management of vasomotor symptoms $(n=1)$. In $40(91 \%)$ of the 44 patients on hormones, the endometrial sampling specimen showed definite evidence of atrophy with hormone effect based on original pathology reports and rereview by our study pathologist. One patient had a sample that was suboptimal to evaluate for hormone effect, and three patients who reported exogenous hormone use at the time of biopsy did not have any evidence of hormone effect on pathology.

The demographics of all patients with atrophy on endometrial sampling are shown in Table 1 and are divided by menopausal status and use of exogenous progestins. Not surprisingly, the average age among the postmenopausal patients was higher than in either premenopausal group. However, the difference in age remained statistically significant $(\mathrm{p}<0.001)$ when comparing only the premenopausal patients on progestins to those not on any hormones, as did the median gravity $(\mathrm{p}=0.005)$ and parity $(\mathrm{p}=0.015)$. The other demographic factors of the premenopausal patients on progestins did not differ significantly from the premenopausal patients not taking hormones. For all of these 29 premenopausal patients not on progestin or combination therapy, the indication for endometrial sampling was abnormal uterine bleeding. Outcomes post-atrophic index biopsy was as follows: four patients underwent hysterectomy for persistent bleeding, one of whom had a malignancy. One patient had a polypectomy with benign final pathology. Seventeen patients experienced resolution of their symptoms. Five of these patients had subsequent endometrial sampling for persistent abnormal uterine bleeding, and all specimens showed either persistent atrophy or weakly proliferative endometrium. Seven patients did not follow up after undergoing initial endometrial sampling.

Figure 1: Study Cohort.

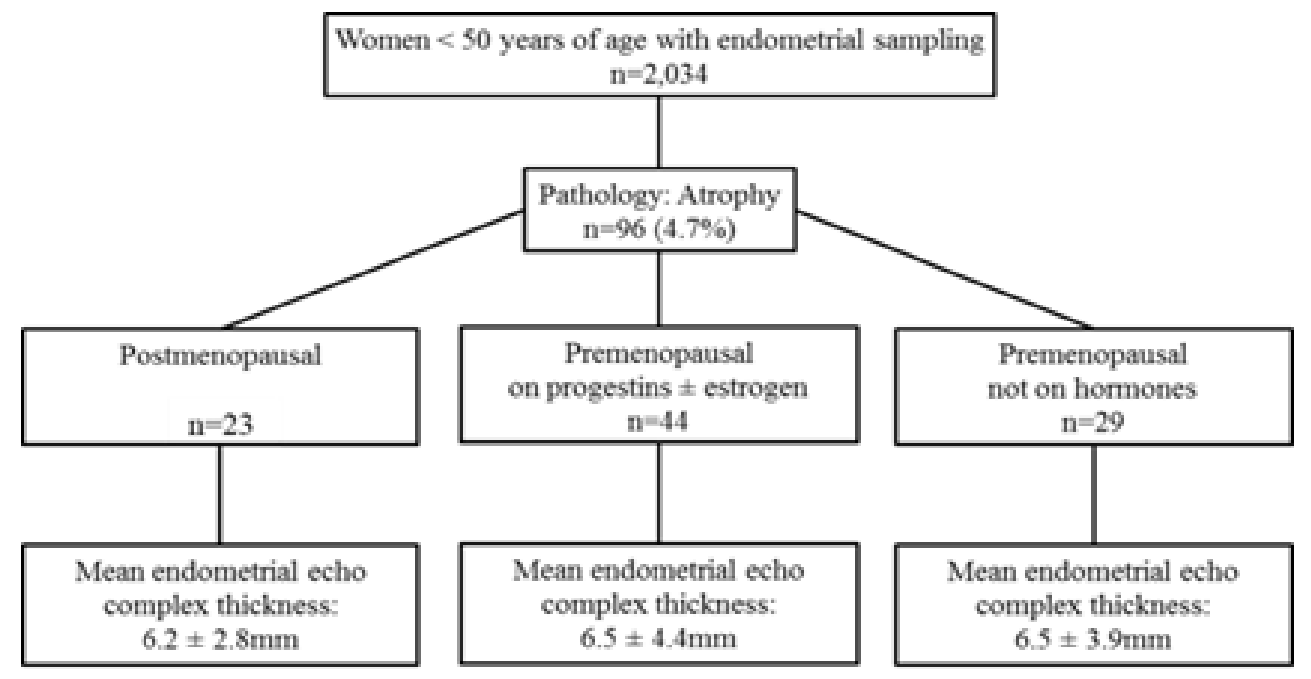

Figure I Study cohort. 
Table I Patient demographics by menopausal state and exogenous progestin or combination hormone use

\begin{tabular}{|c|c|c|c|c|}
\hline \multirow[b]{2}{*}{ Characteristic } & \multicolumn{3}{|c|}{ Premenopausal $(n=73)$} & \multirow[b]{2}{*}{$\mathbf{P}$} \\
\hline & $\begin{array}{l}\text { No Hormones } \\
(n=29)\end{array}$ & $\begin{array}{l}\text { Progestin } \pm \text { Estrogen } \\
(n=44)\end{array}$ & $\begin{array}{l}\text { Postmenopausal } \\
(n=23)\end{array}$ & \\
\hline Age (y) & $43.7 \pm 6.2$ & $37.7 \pm 7.7$ & $46.2 \pm 3.9$ & $<0.001$ \\
\hline Gravity & $4[2,5]$ & $2[0,3]$ & $3[2,4]$ & 0.011 \\
\hline Parity & $3[2,4]$ & $2[0,3]$ & $3[2,3]$ & 0.032 \\
\hline BMI (kg/m2) & $32.2 \pm 8.5$ & $33.0 \pm 7.1$ & $31.3 \pm 9.4$ & 0.802 \\
\hline \multicolumn{5}{|l|}{ Race or Ethnicity } \\
\hline Hispanic & $23(79.3)$ & $32(72.7)$ & $12(52.2)$ & 0.246 \\
\hline White & I (3.4) & $7(15.9)$ & $4(17.4)$ & \\
\hline African American & $2(6.9)$ & $\mathrm{I}(2.3)$ & $3(13.0)$ & \\
\hline Asian & $3(10.3)$ & $2(4.5)$ & $2(8.7)$ & \\
\hline Other & $0(0)$ & $2(4.5)$ & $2(8.7)$ & \\
\hline \multicolumn{5}{|l|}{ Comorbidities } \\
\hline Diabetes Mellitus & $0(0)$ & $5(11.4)$ & $3(13.0)$ & 0.147 \\
\hline Hypertension & $4(13.7)$ & $9(20.5)$ & $6(26.0)$ & 0.537 \\
\hline Thyroid Disease & $2(6.9)$ & $3(6.8)$ & I (4.3) & 0.911 \\
\hline Personal History of Cancer & $2(6.9)$ & $2(4.5)$ & I (4.3) & 0.886 \\
\hline Family History of Cancer & $7(24.1)$ & $5(11.4)$ & $4(17.4)$ & 0.356 \\
\hline
\end{tabular}

BMI, body mass index Data are $\mathrm{n}(\%)$, median (interquartile range $[25 \%$ tile, $75 \%$ tile]), or mean \pm standard deviation unless otherwise specified Seventy-one of the 96 patients underwent transvaginal ultrasound. Twenty-three premenopausal patients were on exogenous progestins alone or in combination at the time of endometrial sampling but not at the time of their ultrasound, and they were excluded from the ultrasound analysis. The mean endometrial echo complex thickness for all patients with atrophy of the endometrium and no other intrauterine pathology was $6.4 \pm 3.6 \mathrm{~mm}$. Among those patients (Figure 1), there was no significant difference $(p=0.97)$ in the mean endometrial echo complex thickness between postmenopausal patients $(6.2 \pm 2.8 \mathrm{~mm})$, premenopausal patients on progestins with or without estrogen $(6.5 \pm 4.4 \mathrm{~mm})$ and premenopausal patients who were not taking exogenous progestins $(6.5 \pm 3.9 \mathrm{~mm})$.

Of the 96 women identified who were younger than 50 years of age and had atrophy on endometrial sampling, 89 (93\%) experienced resolution of their bleeding or had a clinical history compatible with endometrial atrophy, such as the use of exogenous progestins or combination hormone therapy, postmenopausal state, or an ultrasound showing no intracavitary lesions and an endometrial echo complex thickness measuring within 2 standard deviations of the mean for this cohort $(\leq 14 \mathrm{~mm})$. The remaining seven premenopausal patients who were not taking exogenous progestins had ultrasound findings inconsistent with atrophy, such as an intracavitary lesion $(n=4)$, an endometrial echo complex thickness $>14 \mathrm{~mm}(\mathrm{n}=2)$, or an unevaluable endometrial echo complex due to a large uterine mass $(n=1)$.

One $(14 \%)$ of these seven patients was found to have a uterine carcinosarcoma. The patient was a 30 year-old African-American female who presented with abnormal vaginal bleeding. During her initial consult, a pelvic ultrasound revealed a $14 \mathrm{~cm}$ uterus with a thickened endometrial echo complex of $29 \mathrm{~mm}$ and a heterogeneous lower uterine segment. An endometrial biopsy performed on the same day revealed atrophy. She was thus managed with hormonal medication with initial improvement of her bleeding until she returned 7 months later with a $22 \mathrm{~cm}$ uterus, pain, severe anemia and lymphadenopathy. She underwent surgical management and was diagnosed with stage IV carcinosarcoma on final pathology.

\section{Discussion}

In our study, nearly $5 \%$ of women under 50 years of age who underwent endometrial sampling had atrophy. For the majority of patients (67 out of 96) an atrophic endometrium was consistent with clinical history, as they were either postmenopausal or using exogenous progestin or combination hormone therapy. The 29 patients who were premenopausal and not taking progestins or combination therapy all underwent endometrial sampling for a history of abnormal uterine bleeding. On average, they were older and had a higher gravity and parity compared to the group using hormone therapy. These differences are most likely attributable to the high proportion of patients in the progestin or combination hormone group who underwent endometrial sampling for a history of endometrial hyperplasia (45\%) and may have associated infertility or are delaying pregnancy until after resolution of their hyperplasia.

Of the 29 premenopausal patients not taking progestins or combination therapy, seven were identified in whom an atrophic endometrium was inconsistent with clinical and ultrasound findings. These included all patients with a thickened endometrial echo complex $(>14 \mathrm{~mm})$, an unevaluable endometrial echo complex, or an intracavity lesion. Of these patients, one (14\%) was found to have a malignancy. This constellation of clinical factors may place a patient into a higher risk category where the reliability of an atrophic endometrial sampling result may be limited at excluding a uterine malignancy. 
Multiple studies have explored the relationship between preoperative endometrial sampling results and final pathology after hysterectomy. ${ }^{8-11}$ Endometrial biopsies have been shown to have a high overall accuracy in the diagnosis of endometrial cancer, with a sensitivity of $99.6 \%$ in postmenopausal women, but only $91 \%$ in premenopausal women. ${ }^{15,16}$ In addition, the diagnosis is more likely to be missed if the cancer is localized to an endometrial polyp or occupies less than $50 \%$ of the endometrium. ${ }^{17}$ Clark et al. ${ }^{18}$ found that an endometrial biopsy result showing cancer was more accurate at ruling in disease than a negative test result was at ruling it out, with a post test probability of $81.7 \%$ for a positive result and $0.9 \%$ for a negative result.18 Bansal et al reported that the overall ability of preoperative endometrial sampling to detect a malignancy was significantly lower for non-endometrioid histologies with $75 \%$ accuracy for uterine papillary serous and clear cell carcinomas, $59 \%$ for carcinosarcoma, and $52 \%$ for other sarcomas. ${ }^{19}$ Sany et al. ${ }^{20}$ described a correlation between underlying histology and the ability to make an accurate diagnosis based on preoperative endometrial sampling (endometroid carcinoma $78 \%$, non-endometrioid carcinoma $67 \%$, carcinosarcoma $90 \%$, and sarcoma $40 \%) .{ }^{20}$ Given the possibility of false negative results with endometrial sampling, ACOG recommends further testing to rule out endometrial pathology in the setting of persistent abnormal uterine bleeding with benign pathology. ${ }^{2}$ As malignancies of nonendometrioid histology can arise in a background of atrophy and have a higher incidence of being misdiagnosed on endometrial sampling, we propose that women younger than 50 years of age with atrophy on endometrial sampling but with clinical and ultrasonographic findings inconsistent with this result be considered at higher risk of having an underlying malignancy and additional testing should be initiated without further delay.

While transvaginal ultrasound evaluation of the endometrial echo complex thickness using $4 \mathrm{~mm}$ as the cutoff is valuable in postmenopausal women at excluding endometrial hyperplasia and cancer, it is not reliable in premenopausal women. ${ }^{2}$ Normal endometrial echo complex thickness varies based on the patient's menstrual cycle typically ranging from $3-5 \mathrm{~mm}$ at the end of menses to approximately $12-14 \mathrm{~mm}$ in the secretory phase..$^{21,22}$ Based on correlative data of endometrial echo complex thickness and histopathology in premenopausal women with abnormal uterine bleeding published by Ozdemir et al. ${ }^{23}$ the average endometrial echo complex in premenopausal women with atrophy was $4.6 \pm 2.5 \mathrm{~mm} .{ }^{23} \mathrm{In}$ our cohort, the respective mean endometrial echo complex thickness for all patients with atrophy and no intracavitary pathology was $6.4 \pm 3.6 \mathrm{~mm}$. This finding was consistent for all patients with atrophy regardless of menopausal status or any use of exogenous progestins or combination hormone therapy. Based on the above, we used a cutoff for an endometrial echo complex measurement at which the thickness should be considered incompatible with atrophy of $14 \mathrm{~mm}$, which is greater than 2 standard deviations above the mean.

We also propose for further study clinical risk stratification for women younger than 50 years of age with atrophy on endometrial sampling (Table 2) that may help guide clinical management in addition to the integration of other risk factors, such as obesity or family history.

In summary, atrophy is observed in nearly $5 \%$ of women undergoing endometrial sampling under age 50 . Of these, $1 \%$ had an underlying malignancy. On transvaginal ultrasound, the mean endometrial echo complex for patients with atrophy under age 50 is $6.4 \pm 3.6 \mathrm{~mm}$, regardless of menopausal state and hormone use. With only one case of malignancy observed, it is beyond the scope of this study to determine the true incidence of cancer in this population. However, in the setting of abnormal uterine bleeding in this population, we propose to stratify patients into a higher or lower risk category based on menopausal status, exogenous progestin or combination hormone use and ultrasound findings. If a premenopausal woman is not on progestin or combination hormone therapy and ultrasound findings are inconsistent with atrophy, an endometrial sampling result showing atrophy cannot reliably rule out malignancy. We recommend that this subset of patients undergo further testing before malignancy is excluded.

Table 2 Proposed cancer risk stratification for women younger than 50 years of age with atrophy on endometrial sampling

\begin{tabular}{|c|c|}
\hline Risk stratification & Clinical characteristics \\
\hline \multirow{5}{*}{ Low Risk } & Postmenopausal or \\
\hline & Taking exogenous progestins (alone or in combination) or \\
\hline & Pelvic ultrasound findings consistent with atrophic endometrium \\
\hline & Endometrial echo complex $(\leq 14 \mathrm{~mm})$ and \\
\hline & No intracavitary lesions \\
\hline \multirow{7}{*}{ High Risk } & Premenopausal and \\
\hline & No exogenous progestin use and \\
\hline & Pelvic ultrasound findings inconsistent with atrophic endometrium \\
\hline & \\
\hline & Thickened endometrial echo complex $(>\mid 4 \mathrm{~mm})$ and/or \\
\hline & Unevaluable endometrial echo complex and/or \\
\hline & Intracavitary lesion \\
\hline
\end{tabular}




\section{Acknowledgments}

Presented in part at the Western Association of Gynecologic Oncologists Annual Meeting; Huntington Beach, CA; June 27-30, 2012 and the $67^{\text {th }}$ Annual Obstetrical and Gynecological Assembly of SouthernCalifornia; Los Angeles, CA; April 13-14, 2012.

\section{Conflicts of interest}

The authors declare there is no conflict of interests.

\section{References}

1. American College of Obstetricians and Gynecologists. ACOG practice bulletin, clinical management guidelines for obstetrician-gynecologists, number 65, August 2005: management of endometrial cancer. Obstet Gynecol. 2005;106(2):413-425.

2. Committee on Practice Bulletins-Gynecology. Practice bulletin no. 128 : diagnosis of abnormal uterine bleeding in reproductive-aged women. Obstet Gynecol. 2012;120(1):197-206.

3. Gültekin M, Diribaş K, Dursun P, et al. Current management of endometrial hyperplasia and endometrial intraepithelial neoplasia (EIN). Eur J Gynaecol Oncol. 2009;30(4):396-401.

4. Gallos ID, Shehmar M, Thangaratinam S, et al. Oral progestogens vs levonorgestrel-releasing intrauterine system for endometrial hyperplasia: a systematic review and metaanalysis. Am J Obstet Gynecol. 2010;203(6):547.e1-547.e10.

5. Greer BE, Koh WJ, Abu-Rustum N, et al. Uterine Neoplasms. Clinical Practice Guidelines in Oncology. J Natl Compr Canc Netw. 2009;7(5):498-531.

6. Karlsson B, Granberg S, Wikland M, et al. Transvaginal ultrasonography of the endometrium in women with postmenopausal bleeding--a Nordic multicenter study. Am J Obstet Gynecol. 1995;172(5):1488-1494.

7. Zaino RJ. Endometrial atrophy. Interpretation of endometrial biopsies and curettings. Philadelphia, USA: Lipincott-Raven; 1996.

8. Kurman RJ. Morphology of Inactive and Atrophic Endometrium. Blaustein's Pathology of the Female Genital Tract. 5th edn. New York, USA: Springer-Verlag; 2002.

9. McCluggage WG. My approach to the interpretation of endometrial biopsies and curettings. J Clin Pathol. 2006;59(8):801-812.

10. Moyer DL, Felix JC. The effects of progesterone and progestins on endometrial proliferation. Contraception. 1998;57(6):399-403.
11. Rizkalla HF, Higgins M, Kelehan P, et al. Pathological findings associated with the presence of a mirena intrauterine system at hysterectomy. Int $J$ Gynecol Pathol. 2008;27(1):74-78.

12. Felix AS, Weissfeld JL, Stone RA, et al. Factors associated with Type I and Type II endometrial cancer. Cancer Causes Control. 2010;21(11):18511856.

13. Zlatkov V, Radeva V, Cholakova G, et al. Assessment of irregular uterine bleeding in premenopausal women. Akush Ginekol (Sofiia). 2002;41(5):33-35.

14. Sahmay S, Oral E, Saridogan E, et al. Endometrial biopsy findings in infertility: analysis of 12,949 cases. Int J Fertil Menopausal Stud. 1995;40(6):316-321.

15. ACOG Committee on Practice Bulletins--Gynecology. American College of Obstetricians and Gynecologists. ACOG practice bulletin: management of anovulatory bleeding. Int J Gynaecol Obstet. 2001;72(3):263-71.

16. Dijkhuizen FP, Mol BW, Brölmann HA, et al. 2000; The accuracy of endometrial sampling in the diagnosis of patients with endometrial carcinoma and hyperplasia: a meta-analysis. Cancer. 89(8):1765-1772.

17. Guido RS, Kanbour-Shakir A, Rulin MC, et al. Pipelle endometrial sampling. Sensitivity in the detection of endometrial cancer. $J$ Reprod Med. 1995;40(8):553-555.

18. Clark TJ, Mann CH, Shah N, et al. Accuracy of outpatient endometrial biopsy in the diagnosis of endometrial cancer: a systematic quantitative review. BJOG. 2002;109(3):313-321.

19. Bansal N, Herzog TJ, Burke W, et al. The utility of preoperative endometrial sampling for the detection of uterine sarcomas. Gynecol Oncol. 2008;110(1):43-48.

20. Sany O, Singh K, Jha S. Correlation between preoperative endometrial sampling and final endometrial cancer histology. Eur J Gynaecol Oncol. 2012;33(2):142-144.

21. American College of Obstetricians and Gynecologists. ACOG Committee Opinion No. 426: The role of transvaginal ultrasonography in the evaluation of postmenopausal bleeding. Obstet Gynecol. 2009;113(2 Pt $1): 462-464$.

22. Thomas M VVB. Gynecologic Ultrasound. 10th edn. Philadelphia, USA: Lippincott Williams \& Wilkins; 2008.

23. Ozdemir S, Celik C, Gezginç K, et al. Evaluation of endometrial thickness with transvaginal ultrasonography and histopathology in premenopausal women with abnormal vaginal bleeding. Arch Gynecol Obstet. 2010;282(4):395-399. 